\title{
Spatial Visualization of the Heart in Case of Ectopic Beats and Fibrillation
}

\author{
Sándor M. Szilágyi ${ }^{1,2}$, László Szilágyi ${ }^{1,2}$, and Zoltán Benyó \\ ${ }^{1}$ Sapientia - Hungarian Science University of Transylvania, \\ Faculty of Technical and Human Science, Târgu-Mureş, Romania \\ szs@ms.sapientia.ro \\ ${ }^{2}$ Budapest University of Technology and Economics, \\ Dept. of Control Engineering and Information Technology, Budapest, Hungary
}

\begin{abstract}
This paper presents a dynamic heart model based on a parallelized space-time adaptive mesh refinement algorithm (AMRA). The spatial and temporal simulation method of the anisotropic excitable media has to achieve great performance in distributed processing environment. The accuracy and efficiency of the algorithm was tested for anisotropic and inhomogeneous 3D domains using ten Tusscher's and Nygen's cardiac cell models. During propagation of depolarization wave, the kinetic, compositional and rotational anisotrophy is included in the tissue, organ and torso model. The generated inverse ECG with conventional and parallelized algorithm has the same quality, but a speedup of factor 200 can be reached using AMRA modeling and single instruction multiple data (SIMD) programming of the video cards. These results suggest that a powerful personal computer will be able to perform a onesecond long simulation of the spatial electrical dynamics of the heart in approximately five minutes.
\end{abstract}

Keywords: spatial visualization, heart wall movement analysis, parallel processing.

\section{Introduction}

Sudden cardiac death, caused mostly by ventricular fibrillation, is responsible for at least five million deaths in the world each year. Despite decades of research, the mechanisms responsible for ventricular fibrillation are not yet well understood. It would be important to understand how the onset of arrhythmias that cause fibrillation depends on details such as heart's size [15], geometry [1], mechanical and electrical state, anisotropic fiber structure and inhomogeneities [1. The main difficulty in development of a quantitatively accurate simulation of an entire three-dimensional human heart is that the human heart muscle is a strongly excitable medium whose electrical dynamics involve rapidly varying, highly localized fronts [2].

Ectopic heartbeats are arrhythmias involving variations in a normal heartbeat. Sometimes they may occur without obvious cause and are not harmful. However, they are often associated with electrolyte abnormalities in the blood

D. Mery and L. Rueda (Eds.): PSIVT 2007, LNCS 4872, pp. 548 561, 2007.

(C) Springer-Verlag Berlin Heidelberg 2007 
that should be treated. Many times ectopic beats can be associated with ischemia, or local reduction in blood supply to the heart. Once an ectopic beat appears, the underlying reversible reasons should be investigated, even if no further treatment is needed.

An important aspect of ectopic beats caused by the altered depolarization of cardiac tissue is the significantly altered displacement of the heart during the whole beat. This special movement is easily visible in echocardiography image sequences. Each ectopic beat has a patient dependent special waveform caused by the irregular depolarization order of the cardiac tissue. The formulation of an ectopic beat and the generated mechanical movement can be simulated with computers.

In ventricular tissues the width of a depolarization front is usually less than half $\mathrm{mm}$. A simulation approximating the dynamics of such a front requires a spatial resolution of $\Delta x \leq 0.1 \mathrm{~mm}$. Forasmuch the muscle in an adult heart has a volume of $250 \mathrm{~cm}^{3}$, and so a uniform spatial representation require at least $2.5 \cdot 10^{8}$ nodes. Taking into account that each node's state is described with at least 50 floating numbers, the necessary storage space rises higher than $50 G B$, which exceeds by far the available memory of personal computers. The rapid depolarization of the cell membrane is the fastest event in the heart; it blows over in few hundred microseconds, which implies a time step $\Delta t \leq 25 \mu \mathrm{s}$. Since dangerous arrhythmias may require several seconds to become established, the $10^{10}$ floating point numbers associated with the spatial representation would have to be evolved over $10^{5}-10^{6}$ time steps. Such a huge uniform mesh calculation currently exceeds all existing computational resources [3].

The spatiotemporal structure of wave dynamics in excitable media suggests an automatically adjustable resolution in time and space. The basic idea of this improvement [23] is deducted from experiments and simulations [4, which recommend that the function of electrical membrane potential of a ventricular cell $f_{V}(t, x, y, z)$ in the fibrillating state consists of many spirals or of many scroll waves. An interesting property of these spatiotemporal disordered states is that the dynamics is sparse: at any given moment, only a small volume fraction of the excitable medium is depolarized by the fronts, and away from them, the dynamics is slowly varying in space and time. This idea permits the decrement of necessary computational effort and storage space for regular beats but the total front volume can greatly increase with fibrillating state. By varying the spatiotemporal resolution to concentrate computational effort primarily along the areas with large spatial and temporal gradients, it is possible to reduce the computational load and memory needs by orders of magnitude.

The rest of the paper describes the applied human cell and tissue model, the time and spatial position dependent heart and torso model, the position of the ectopic beat generators, the adaptively variable resolution wave-propagation method and the parallel processing of these algorithms aided by graphic cards. Using this algorithm, we can simulate the electric and mechanic formulation of ectopic beats on a parallel functioning platform. 


\section{Materials and Methods}

\subsection{Human Cell and Tissue Model}

We used the ten Tusscher heart cell model 14 for ventricular and Nygren's model 9 for atrial cells, to investigate the accuracy and efficiency of the simulation algorithm. These models are based on recent experimental data on most of the major ionic currents, such as the fast sodium, L-type calcium, transient outward, rapid and slow delayed rectifier, and inward rectifier currents. With the inclusion of basic calcium dynamics, the contraction and restitution mechanism of the muscle cells can be investigated. The model is able to reproduce human epicardial, endocardial and $\mathrm{M}$ cell action potentials, to modify the internal state of the cells and to show that differences can be explained by differences in the transient outward and slow delayed rectifier currents. These properties allow us to study the evolution of reentrant arrhythmias. The conduction velocity restitution of this model is broader than in other models and agrees better with the available data. We conclude that the applied model can reproduce a variety of electrophysiological behaviors and provides a basis for studies of reentrant arrhythmias in human heart tissue.

As described in 14, the cell membrane can be modeled as a capacitor connected in parallel with variable resistances and batteries representing the different ionic currents and pumps. The electrophysiological behavior of a single cell is described as:

$$
\frac{d V}{d t}=-\frac{I_{\text {ion }}+I_{\text {stim }}}{C_{\text {memb }}},
$$

where $V$ is the voltage, $t$ is time, $I_{\text {ion }}$ is the sum of all transmembrane ionic currents, $I_{\text {stim }}$ is the externally applied stimulus current, and $C_{\text {memb }}$ is the cell capacitance per unit surface area.

The ionic current is given as the following sum:

$$
\begin{aligned}
I_{\text {ion }} & =I_{N a}+I_{K 1}+I_{t o}+I_{K r}+I_{K s}+I_{C a L}+I_{N a C a}+ \\
& +I_{N a K}+I_{p C a}+I_{p K}+I_{b C a}+I_{b K}
\end{aligned}
$$

where $I_{\mathrm{NaCa}}$ is $\mathrm{Na}^{+} / \mathrm{Ca}^{2+}$ exchanger current, $I_{\mathrm{NaK}}$ is $\mathrm{Na}^{+} / \mathrm{K}^{+}$pump current, $I_{p C a}$ and $I_{p K}$ are plateau-, $I_{b C a}$ and $I_{b k}$ are background- $C a^{2+}$ and $K^{+}$currents. The fast $\mathrm{Na}^{+}$current that is responsible for the fast depolarization of the cardiac cells, is formulated by:

$$
I_{N a}=G_{N a} \cdot m^{3} \cdot h \cdot j \cdot\left(V-E_{N a}\right),
$$

where $G_{N a}$ is the sodium conductance, $m$ represents the activation gate, $h$ is the fast and $j$ the slow inactivation gate. All detailed equations are described in 8 . These gates have mainly a voltage dependent behavior. The maximal value of the first derivative of the L-type calcium current $I_{C a L}$, transient outward current $I_{t o}$, slow delayed rectifier current $I_{K s}$, rapid delayed rectifier current $I_{K r}$, and inward rectifier $K^{+}$current $I_{K 1}$, and all other described currents are lower with at least two magnitudes than for the fast $\mathrm{Na}^{+}$current $I_{\mathrm{Na}}$. 
A homogenous spatial cardiac tissue can be modeled in space as a continuous system, using the following partial differential equation:

$$
\frac{d V}{d t}=\frac{1}{C_{\mathrm{memb}}}\left(-I_{\mathrm{ion}}-I_{\mathrm{stim}}+\frac{1}{\rho_{x} S_{x}} \frac{\partial^{2} V}{\partial x^{2}}+\frac{1}{\rho_{y} S_{y}} \frac{\partial^{2} V}{\partial y^{2}}+\frac{1}{\rho_{z} S_{z}} \frac{\partial^{2} V}{\partial z^{2}}\right)
$$

where $\rho_{x}, \rho_{y}, \rho_{z}$, are the cellular resistivity and $S_{x}, S_{y}, S_{z}$, are the surface-tovolume ratio in the $x, y$ and $z$ directions.

Computational modeling of the cardiac tissue is a useful tool for developing mechanistic insights into cardiac dynamics. The most important parts of human cardiac analysis are atria and ventricular tissue modeling. In this study, the tissue-level excitation mechanism is based on Fast's work [6]. In this stage, each tissue element works as a secondary generator element. These elements can generate a depolarization wave if the adjacent elements are repolarized; otherwise, the wave propagation is swooned.

Our study uses Harrild's atria model [8] that is the first membrane-based description of spatial conduction in a realistic human atrial geometry. This model includes both the left and right atria, including representations of the major atrial bundles and a right-sided endocardial network of pectinate muscle. The membrane's kinetics is governed by the Nygren [9] formulation for the human atrial cell. An advantage of this model is that it provides an easy perceptibility of atrial activation, particularly in regions that cannot be easily recorded in patients.

It has long been appreciated that cardiac ventricular fibers are arranged as counter-wound helices encircling the ventricular cavities, and that the orientation of these fibers depends on transmural location. Fibers tend to lie in planes parallel to the epicardium, approaching a longitudinal orientation on the ventricular surfaces, and rotating toward the horizontal near the mid-wall. The direct anatomical reconstructions are labor-intensive and time-consuming tasks. In our study, we applied Winslow's ventricular tissue model [17].

\subsection{Heart and Torso Model}

There are many possible different heart structures [10]. To describe various representative cases, we studied our breast MRI records (42 examples) and numerous CT images. These samples lead us to construct a morphological heart structure for simulation, using a segmentation method presented in [5]. The obtained results were classified by physiologists and used to identify each atrial and ventricular region. The identification process uses as a base Harrild's atria model [8] and Winslow's ventricular tissue model [17.

From the correctly segmented images, we constructed a spatial representation of the heart, using an averaging technique. Such a prototype heart representation must be adjusted taking into consideration the ECG data. The ECG has an important role, as it may describe the electric property of the heart. For example, the mechanic related data obtained from MRI and CT images cannot give us any information about some malfunctions, such as the presence of numerous ectopic beats. An ultrasound image sequence, due to the relation between 
electric and mechanic properties of the heart, may hold some mechanic information that can be used to identify diverse electric dysfunctions. The obtained heart model prototype contains most mechanic characteristics of the heart, such as tissue mass, wall thickness, internal structure of atria and ventricles. Some electric properties, such as conduction speed of the depolarization wave, are not deductible from captured images and the unique information sources are the parameters determined from the ECG signal. For example, the activation delay between atria and ventricles can be determined from $\mathrm{P}$ and $\mathrm{R}$ wave distance. The increment speed of the $\mathrm{R}$ wave determines the conduction speed on the ventricular tissue. This information was used to construct the electric-mechanic heart model as described in [12.

The anatomical structure of the atria 8 and ventricles 13 was involved in the geometrical model of the heart and torso. The torso, lung, endo- and epicardial surfaces were initially divided into 23647, 38844, 78809 and 89723 sub-units. For each of the units, the constant properties were determined (mass, tissue type but not the tissue state). During an ordinary simulation, the number of these sub-units can vary by demand. The only restriction relies on preserving the ratio among the numbers of sub-units pre-determined for each heart region. Such a heart model could have a maximal spatial resolution of $0.025 \mathrm{~mm}$ (restricted by the size of computer main memory) that means more than ten billion individual compartments at highest decomposition. To allow a flexible simulation, we may choose the minimal time-slice between $0.01 \mathrm{~ms}$ and $2 \mathrm{~ms}$. Each of these units may contain diverse variable properties, such as tissue state, ionic concentrations or diverse malfunction information such as ischemia. Starting from anatomical information and selected resolution for both time and space, the heart is constructed using tetra meshes. During a simulation with selected spatial resolution, the number of meshes remains constant. However, the mechanical displacement of the heart modifies the shape of each created mesh structure.

The structure of the torso, its spatial position, the relative position and distance of the compartments with respect to the electrodes, and the electrical behavior of the torso's contents are necessary to be known. As the model has to take in consideration extremely numerous parameter values, the problem cannot be solved in a deterministic way (we have much more unknown values than known equations). That is why a stochastic method (genetic algorithm, adaptive neural networks and fuzzy systems) should be applied to determine the values of the parameters. The search space of the optimization problem was reduced using the genetic algorithm (GA) presented in [7].

\subsection{Mathematical Description of the Compartments}

The heart is represented as a set of finite homogenous elements, called compartments. Since their size is obviously much larger than that of actual biological cells, these units effectively represent small tetrahedron-shaped groups of biological cells, and must capture their macroscopic behavior rather than the microscopic behavior of individual cells. Microscopic inter/intracellular interactions, such as ionic flow across membrane boundaries, were described in the cell 
model presentation. Compartment connectedness was defined as the set of rules that establish which units are considered directly connected to a given one, for the purposes of electrophysiological simulation, such that myocardial activation may be directly propagated between them. These rules are based on atria and ventricles anatomy; they define the neighborhood for each unit.

Each compartment was considered homogenous, constructed by only one type of tissue with well-defined properties, such as cell type, cell state, cell activation potential (AP) function. The type of cells determines the electrical propagation properties, but no additional considerations were taken in, such as tissue fiber torsion and so on. The environmental parameters such as $4 \mathrm{D}$ position ( $x$, $y, z$ spatial coordinates and time), conduction speed of stimulus, weight and connection with neighbor structures, localize each unit.

The heart behavior is characterized by the following parameters:

1. Type of cells: $T$ (such as ventricular muscle cell or Purkinje fiber cell);

2. State (time varying): $S$ (normal, ischemia);

3. Function of activation potential variation: $A P(T, S, t)$ (each compartment has a specific activation potential function that depends from cell type and state);

4. Space position in time: $\operatorname{Pos}_{C}(x, y, z, t)$ (in every moment a given compartment has a spatial position);

5. Conduction speed of the stimulus: $C S(T, S)$ (it is type and state dependent);

6. Weight of the contents of the compartment: $M$;

7. Connections with other compartments;

8. The position of the electrode: $\operatorname{Pos}_{E}(x, y, z, t)$ (the measuring electrode has a time dependent spatial position);

9. The relative resistance of the electrode: $R_{E, C}\left(\operatorname{Pos}_{C}, \operatorname{Pos}_{E}\right)$ (the time dependent electric resistance of the human tissue from the studied compartment to a given electrode).

Because the main ion channels situated inside the cells have a quite complicated behavior (with lots of unknown parameters), the activation potential function of the compartment was considered as basic input parameter (we determine an AP function - based on cell model - with static shape for each cell type and state). Due to contractions of the heart, respiration, and other disturbing phenomena, the position of compartments was considered time varying. The mathematical expressions presented in the followings, that describe compartment behavior are time variant.

Let $V_{C}$ be the potential of an arbitrary compartment $C: V_{C}(t)=A P(T, S, t-$ $\tau_{C}$ ), where $\tau_{C}$ is the time the stimulus needs to reach compartment $C$. The activation potential function that varies from cell type $T$ and state $S$, has a short delay $\tau_{C}$ due to activation propagation until compartment $C$.

The measured potential $E_{j}$, generated by compartment $C_{i}$ is:

$$
E_{j, C i}(t)=V_{C i}(t) \cdot R_{E j, C i}(t)-E_{\mathrm{GND}, C i}(t),
$$

where $R_{E j, C i}(t)$ represents the time varying resistance from compartment $C_{i}$ to electrode $E_{j}$. Using bipolar electrodes, the value measured on the reference 
electrode $E_{\mathrm{GND}}$ will be subtracted. As all compartments have an accession to the measured potential on each electrode, the measured voltage on electrode $E_{j}$ will become the sum of each $E_{j, C i}(t)$ generated by compartment $C_{i}$ :

$$
E_{j}(t)=\sum_{i=0}^{N-1}\left[V_{C i}(t) \cdot R_{E j, C i(t)}-E_{\mathrm{GND}, C i}(t)\right],
$$

where $N$ is the number of compartments. These equations determine the measured electrical potentials and the inner mechanism in the heart. During the simulation, these voltages were determined for each compartment and electrode for every time-slice (mostly between $0.1 \mathrm{~ms}$ and $2 \mathrm{~ms}$ depending from the phase of AP function).

\subsection{Connections Between Electric and Mechanic Properties}

The time-varying evolution of the cardiac volume is determined by the interconnection of electrical and mechanical phenomena. In a whole cardiac cycle there are two extremity values. The maximal volume can be coupled with the starting moment of ventricular contraction. The depolarization wave normally starts from the sino-atrial node (SA) and propagates through the atrioventricular node $(\mathrm{AV})$ and ventricles. The moment of minimal volume shortly precedes the termination of ventricular contraction, but is much more difficult to identify, due to the dead time of a normal cardiac cell. This delay is caused by the strange property of a regular cardiac cell, whose electric response is most directly caused by the depolarization wave (fast $\mathrm{Na}^{+}$channels), but the mechanical contraction is controlled by the much slower $\mathrm{Ca}^{2+}$ channels. The calcium channel opens at $10-20 \mathrm{~ms}$ after depolarization, and the maximal contraction follows in about $80 \mathrm{~ms}$ [16.

\subsection{Adaptively Varied Resolution}

As presented earlier, the simulation of each compartment at each small timeslice needs a powerful computer. To enhance the simulation performance we can increase the computational power of the simulation platform and modify the algorithm such a way, that it determines the most important data more accurately. Anyway, due to the limited computational power of the computer, the simulation must contain approximations. In our formulation, the simulation task can be performed in the following manners:

- determine a pre-defined time and space resolution (not adaptive);

- guarantee an estimation error that is lower than a pre-defined threshold value (adaptive);

- guarantee a pre-defined processing speed (adaptive).

In the first case nothing in know about the simulation speed and estimation error. The simulation algorithm uses pre-defined time and space resolution and the result's performance (speed and accuracy) can only be estimated. The second processing manner has an adaptive behavior. Resolution is not important, 
but the estimation error is. This approximation of the problem leads to low estimation error, but we have no guaranteed processing speed. The processing speed may highly depend on the heart's state (see Discussions section for details). The third approximation of the problem is useful to create an on-line processing system. However, in this situation, we have the pre-defined simulation speed, but we do not have any control regarding simulation accuracy. In both adaptive approximations of the problem, the scalability of the simulation is realized in the same manner.

During the simulation problem, the key element is the compartment. Each compartment has a time dependent voltage that is increased by depolarization waves and decreased due the self-repolarization process. Both high voltage increment and a high diversity of the compartments (adjacent compartments can have significantly different voltage levels) increases estimation error. This error is estimated by the following formula:

$$
\operatorname{err}(C, t)=\lambda_{d} \frac{d V_{C}}{d t}+\lambda_{v} \sum_{i=0}^{N-1}\left\{\lambda_{C, C_{i}}(t) \cdot\left[V_{C_{i}}(t)-V_{C}(t)\right]\right\}^{2} .
$$

The estimation error is weighted by $\lambda_{d}$ (derivative weight) and $\lambda_{v}$ (voltage weight). The derivative term contains the voltage's increment caused by the fast $\mathrm{Na}^{+}$ionic current during depolarization and by $\mathrm{Ca}^{2+}$ and $\mathrm{K}^{+}$currents during repolarization phase. In the second term, $\lambda_{C, C_{i}}$ represents a weight between compartments $C$ and $C_{i}$. This weight is considered time dependent, as the distance among compartments may vary during simulation. A high voltage difference may increase estimation errors dramatically. From this formula emerges, that the most sensitive moments are the moment of depolarization especially in presence of multiple depolarizing fronts. From the determined estimation error, its variance in time, and the initial settings referring to error threshold or simulation speed, the necessary time and space resolutions are determined. However, during a whole heartbeat the estimation error may vary, that implies the spatial and temporal modification of the used resolution. In order to assure a good alignment among diversely selected resolution slices, each high-resolution value must be selected $2^{i}$ times shorter than the initial reference resolution (this is the coarsest resolution in both time and space). To assure proper resolution values, each determined variable is rounded down to the closest allowed level. Data on all resolution levels are synchronized only after one full time step on the coarsest grid level is completed.

The efficiency of the method arises from its ability to refine or to coarsen the spatial and temporal representations of sub-units automatically and locally. The approximation errors are estimated on each sub-units to determinate the lowest necessary resolution to keep under a pre-defined tolerance value.

The most important factor that demands a finer temporal and spatial resolution to keep the estimation errors under the pre-determined tolerance level, is the fast depolarization wave of the atrial and ventricular tissue cells. The simulation program varies the resolution in concordance with the first derivative of the activation potential. 


\subsection{Parallel Processing}

The implementation of the method allows high parallelization. As sub-unit potential values are determined independently from each other at all possible resolution levels, these tasks can be processed on separate processors with reduced communication needs. The hardware accelerated programmability of graphical processing units (GPUs) (that may contain up to 320 individual processor units) admits the development of programs called shaders vertex and fragment shaders, which are loaded into the graphics card memory for replacing the fixed functionality. The fragment shaders are used in our method to perform the SIMD commands for each sub-unit. From architectural concepts results that the GPUs are most efficient in case of more than 1000 similar tasks, which is caused by the relatively long video memory delay.

\subsection{Simulation Platforms}

Experiments were performed on four platforms with different configuration shown in Table 1. Each computer has $1 G B$ memory that admits a maximal resolution between $0.01 \mathrm{~mm}$ (normal beats) and $0.1 \mathrm{~mm}$ (ventricular fibrillation) for the most critical area (highly restricted size). The program was developed in $\mathrm{C}++$ programming environment and the shader programs were developed using ATI's graph library (shader model 3.0).

\section{Results}

Table 2 informs about simulation times using various configurations. All simulation tasks were performed with adaptive time and spatial resolution, and Table 2 show the finest ones among them. The simulated normal and pathological cases have one second duration. In all cases, the number of simultaneously performable tasks has the order of thousands. The conventional simulation (constant resolution) was performed only for $1 \mathrm{~mm}$ spatial and $0.2 \mathrm{~ms}$ temporal units, and was slower about 200 times in normal and 35 times in fibrillating case.

Table 1. Configuration of the simulation platforms involved in this study

\begin{tabular}{llll}
\hline Configuration & CPU & GPU & RAM \\
\hline 1st & Athlon 3000+ & nVidia 6600 & $1 G B$ DDR \\
2nd & Core2 Duo 6400 & ATI 1950 Pro & $1 G B$ DDR2 \\
3rd & Core2 Duo 6400 & $2 \times$ ATI 1950 Pro & $1 G B$ DDR2 \\
4th & Pentium D805 & nVidia 7600GT & $1 G B$ DDR2 \\
\hline
\end{tabular}

Figure 1(a) elucidates the relation between estimation error and spatial resolution. A lower spatial resolution increases the estimation error of the depolarization wave. The obtained results are almost similar for pathological cases. The propagation of the depolarization wave for an anisotropic tissue is presented in 
Table 2. The whole simulation and visualization time of a one second duration event performed on involved platforms using two spatial and temporal resolutions

\begin{tabular}{|c|c|c|c|}
\hline $\begin{array}{l}\text { Configuration and } \\
\text { Resolution }\end{array}$ & $\begin{array}{l}\text { Normal } \\
\text { beat }\end{array}$ & $\begin{array}{l}\text { Ectopic } \\
\text { beat }\end{array}$ & $\begin{array}{l}\text { Ventricular } \\
\text { fibrillation }\end{array}$ \\
\hline $1 \mathrm{st}-(1 \mathrm{~mm}, 0.2 \mathrm{~ms})$ & $11.3 \mathrm{~s}$ & $37.15 \mathrm{~s}$ & $4 \min 2 s$ \\
\hline $2 \mathrm{nd}-(1 \mathrm{~mm}, 0.2 \mathrm{~ms})$ & $1.32 \mathrm{~s}$ & $5.21 \mathrm{~s}$ & $33.11 s$ \\
\hline $3 \mathrm{rd}-(1 \mathrm{~mm}, 0.2 \mathrm{~ms})$ & $0.7 \mathrm{~s}$ & $2.68 s$ & $17.13 \mathrm{~s}$ \\
\hline 4 th $-(1 m m, 0.2 m s)$ & $2.37 \mathrm{~s}$ & $9.03 s$ & $57.48 s$ \\
\hline $1 \mathrm{st}-(0.1 \mathrm{~mm}, 0.05 \mathrm{~ms})$ & $1 \mathrm{~h} 11 \mathrm{~min}$ & $4 h 22 \min$ & $29 \mathrm{~h} 11 \mathrm{~min}$ \\
\hline $2 \mathrm{nd}-(0.1 \mathrm{~mm}, 0.05 \mathrm{~ms})$ & $9 \min 20 s$ & $37 \mathrm{~min} 10 \mathrm{~s}$ & $3 h 53 \mathrm{~min}$ \\
\hline $3 \mathrm{rd}-(0.1 \mathrm{~mm}, 0.05 \mathrm{~ms})$ & $5 \min 3 s$ & $19 \mathrm{~min} 17 \mathrm{~s}$ & $1 h 59 \min$ \\
\hline 4 th $-(0.1 \mathrm{~mm}, 0.05 \mathrm{~ms})$ & $15 \mathrm{~min} 40 \mathrm{~s}$ & $1 h 6 \mathrm{~min}$ & $6 h 42 \mathrm{~min}$ \\
\hline
\end{tabular}

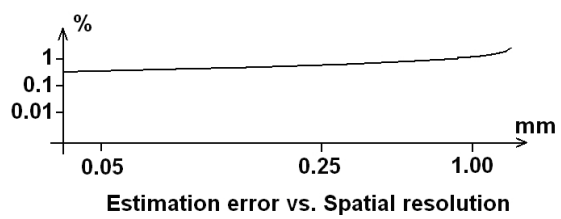

(a)

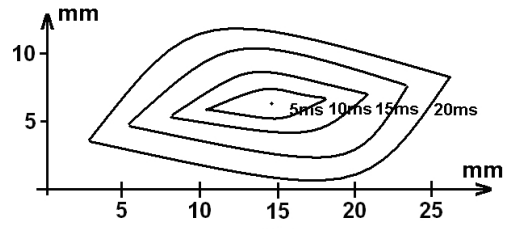

(b)

Fig. 1. (a) The estimation error plotted against the chosen spatial resolution in case of a normal beat, (b) The simulated depolarization wave in anisotropic ventricular tissue: from the pace origin, the wave front propagation is plotted with $5 \mathrm{~ms}$ resolution
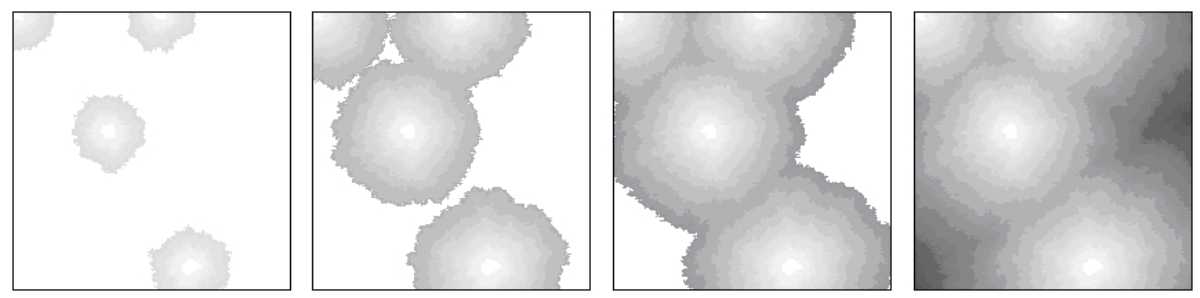

Fig. 2. Propagation of the depolarization wave in a ventricular tissue area during ventricular fibrillation. The visualized area contains four ectopic points. The white excitable area is fast depolarized and the arisen wave fronts extinguish each other. The gray level of each point represents the voltage level for each individual cell. Each image represents a $50 \mathrm{~mm}$ wide square; time step between neighbor squares is $10 \mathrm{~ms}$.

Fig. 1(b). Figure 2 presents the collision of the depolarization waves. The depolarization of various ventricular slices for normal case is presented in Fig. 3 . The simulated ECG signal in normal and abnormal case (ventricular hypertrophy) can be seen in Fig. 4. The spatial representation of the ventricles during a normal heart beat is presented in Fig. 5. The resting and contracting tissue 


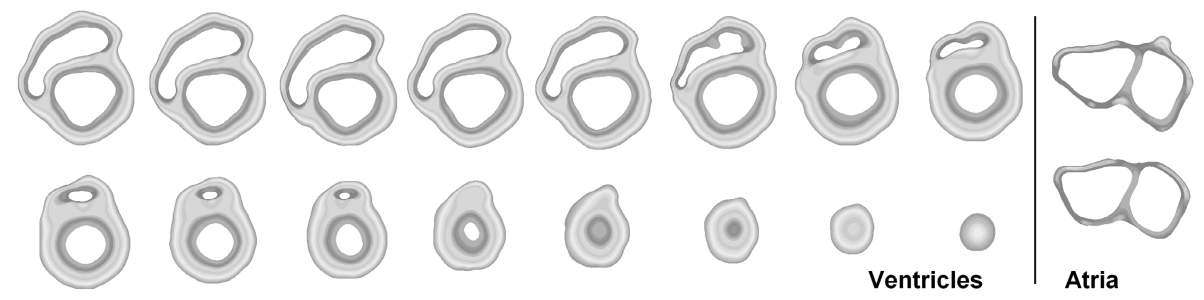

Fig. 3. The propagation of the depolarization wave in the ventricular and atrial tissue. In the left side of the image the consecutive ventricular slices are presented from the ventricular top region to apex (using $5 \mathrm{~mm}$ distance among consecutive slices). The propagation of the depolarization wave is presented, simulating a normal heart beat. The right-sided two images present atrial slices ( $5 \mathrm{~mm}$ distance).

is visible in the first and second rows, respectively. In this simulation, a $0.2 \mathrm{~mm}$ spatial resolution was used.

\section{Discussion and Conclusion}

Table 1 presents four configurations with shader model (SM) 3.0 ready GPUs. The 3rd configuration is the most powerful one, due to the cross-fire connected ATI 1950 PROs. The type of CPU (Intel or AMD), the clock speed (1.86 $2.66 G H z$ ), the core number (solo or duo) and memory bandwidth (DDR or DDR2) did not play an important role because a powerful video card has a much higher floating-point calculation power (internally has 8-36 pixel shader units). In all cases, the size of memory was selected at $1 G B$ that restricts the applicable maximal resolution.

Table 2 summarizes a simulation for normal beat, ectopic beat and ventricular fibrillation state. The finest spatial and temporal resolution was 16 times greater in case of normal beat, 32 times greater in case of Ectopic beat and 64 times greater in case of ventricular fibrillation. This result is in perfect concordance with the complexity of the studied events. A more complex event implies longer depolarization waveform that enforces the processing algorithm to choose lower spatial and temporal steps.

From the data of Table 2 we could observe the clear dominance of GPUs. Although the spatial and temporal resolution limit the necessary simulation time, in all cases a massive parallelization could be performed. All shader programs were created using a low-level programming environment. We could observe that in normal cases, the active depolarization wave front has a much lower size than in case of ventricular beat of ventricular fibrillation. In a complex biological situation, as the wave front size grows, the parallelization becomes harder. This assumption is reflected by the simulation times from Table 2. It is observable that a normal heart has at least 20 times lower front area than a fibrillating one. As a cardiac muscle (especially left ventricular), become less homogeneous, the relative simulating speed decreases. Some basic characteristics of the heart such 


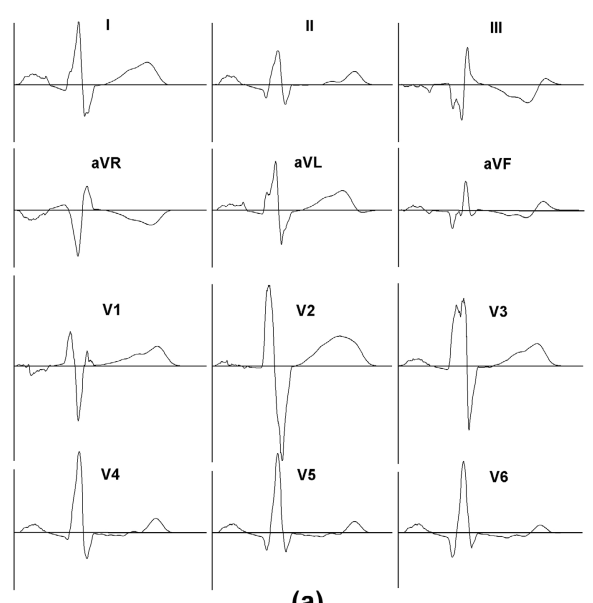

(a)

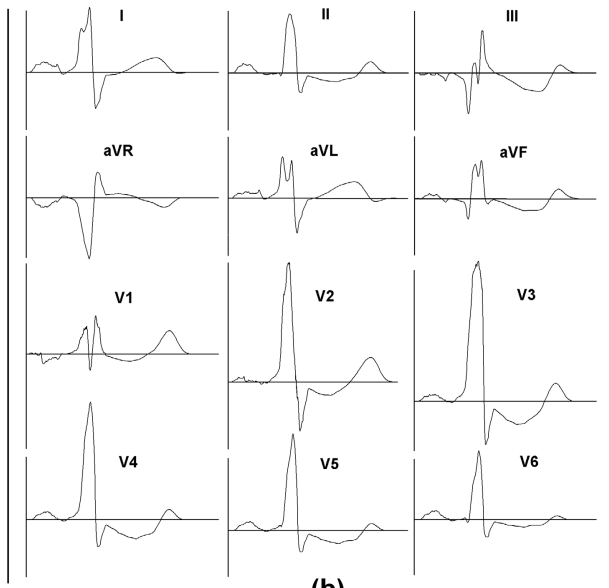

(b)

Fig. 4. Simulated ECG signal in: (a) normal case, and (b) abnormal case (presence of accessory pathway)

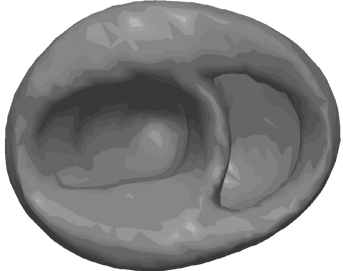

(a)

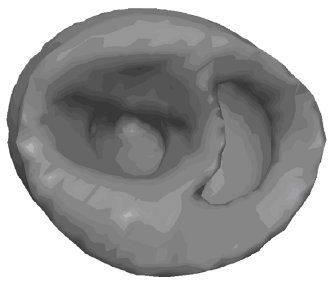

(d)

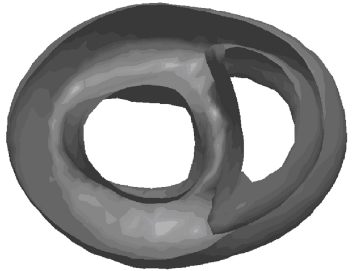

(b)

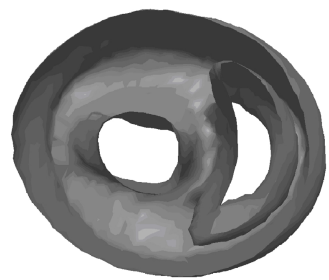

(e)

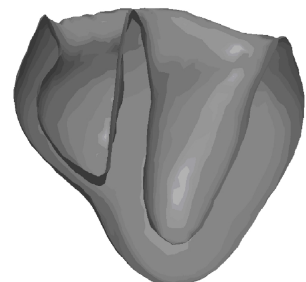

(c)

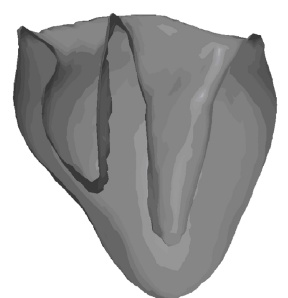

(f)

Fig. 5. The spatial representation of the ventricles in resting ((a), (b) and (c) images) and contracted ((d), (e) and (f) images) state during a normal beat as follows: (a) and (d) upper view, (b) and (e) sectioned bottom view, (c) and (f) sectioned frontal view)

as size, maximal tissue volume and left wall width, significantly influence the maximal performance.

Figure 1(a) represents the estimation error in function of spatial resolution. The temporal resolution has almost similar effect, but with lower impact. From 
measurements, we could deduct that estimation error is free from physiological state. In normal and pathological cases, we measured almost the same error values.

In this paper, we have discussed new features and new capabilities of a space-time adaptive heart-modeling algorithm. We have shown the algorithm's ability to simulate inhomogeneous and strongly anisotropic tissue regions (see Fig. 11(b)).

This method can provide a variety of advances in addition to reductions in time and memory requirements. For example, the algorithm allows a more complex ionic model, higher spatial resolution of a non-linear tissue model. Similarly, it allows the use of higher spatial and temporal resolution to reduce the angle dependence of propagation patterns in spatial domains with rotational anisotropy or to verify that a calculation is sufficiently resolved, so that an increase in resolution does not affect the results (see Fig. 1).

From Fig. 2 we can conclude that the diverse depolarizing wave fronts are unifying and the arisen wave fronts extinguish each other. The simulation was done on a simple ventricular tissue surface to be able to verify the obtained results and to compare with other simulation methods, such as presented in [2. We can affirm that the obtained front shapes were almost the same.

The propagation of the depolarization wave in the ventricular and atrial tissue is presented in Fig. 3. The propagation of the depolarization wave can be seen in the consecutive slices. Using this view, we can supervise the propagation of the depolarizing waves in various circumstances, such as normal beat, ectopic beat, Wolff-Parkinson-White syndrome and ventricular fibrillation.

Besides the wave propagation, the simulated ECG can be visualized (see Fig. 4). The simulation model combined with a forward heart model presented in [12] can yield a simulated ECG.

It is important to study the shape of the heart during a whole cycle. Despite various perturbing phenomena, it was possible to realize the spatial representation of the heart or some segments of it (see Fig. 5). Using this kind of approach, we can balance between performance and accuracy. The optimal solution may depend on the used platform, studied events and the available time.

We have presented a massively parallelized flexible and efficient heart simulation method that uses almost all features of a modern processing hardware. After that, we have demonstrated that the processor of a modern graphics card can provide better performance than a modern CPU under certain conditions, in particular, allocating data in a regular and parallel manner. In these situations, the GPU should operate in a SIMD fashion to get the most performance hit. Experimental results show that the graphics card can be exploited in order to perform non-rendering tasks.

Acknowledgements. This research was supported by the Hungarian National Research Funds (OTKA) under Grant No. T069055, Sapientia Institute for Research Programmes and the Communitas Foundation. 


\section{References}

1. Antzelevitch, C., Shimizu, W., Yan, G.-X., Sicouri, S., Weissenburger, J., Nesterenko, V.V., Burashnikov, A., Di Diego, J., Saffitz, J., Thomas, G.P.: The $\mathrm{M}$ cell: Its contribution to the ECG and to normal and abnormal electrical function of the heart. J. Cardiovasc. Electrophysiol. 10, 1124-1152 (1999)

2. Cherry, E.M., Greenside, H.S., Henriquez, C.S.: A Space-Time Adaptive Method for Simulating Complex Cardiac Dynamics. Phys. Rev. Lett. 84, 1343-1346 (2000)

3. Cherry, E.M., Greenside, H.S., Henriquez, C.S.: Efficient simulation of threedimensional anisotropic cardiac tissue using an adaptive mesh refinement method. Chaos 13, 853-865 (2003)

4. Courtemanche, M.: Complex spiral wave dynamics in a spatially distributed ionic model of cardiac electrical activity. Chaos 6, 579-600 (1996)

5. Dumoulin, S.O., Hoge, R.D., Baker Jr., C.L., Hess, R.F., Achtman, R.L., Evans, A.C.: Automatic volumetric segmentation of human visual retinotopic cortex. Neuroimage $18,576-587$ (2003)

6. Fast, V.G., Rohr, S., Gillis, A.M., Kléber, A.G.: Activation of Cardiac Tissue by Extracellular Electrical Shocks: Formation of 'Secondary Sources' at Intercellular Clefts in Monolayers of Cultured Myocytes. Circ. Res. 82, 375-385 (1998)

7. Godefroid, P., Khurshid, S.: Exploring Very Large State Spaces Using Genetic Algorithms. In: Katoen, J.-P., Stevens, P. (eds.) ETAPS 2002 and TACAS 2002. LNCS, vol. 2280, pp. 266-280. Springer, Heidelberg (2002)

8. Harrild, D.M., Henriquez, C.S.: A Computer Model of Normal Conduction in the Human Atria. Circul. Res. 87, 25-36 (2000)

9. Nygren, A., Fiset, C., Firek, L., Clark, J.W., Lindblad, D.S., Clark, R.B., Giles, W.R.: Mathematical Model of an Adult Human Atria Cell: The Role of K+ Currents in Repolarization. Circul. Res. 82, 63-81 (1998)

10. Quan, W., Evans, S.J.: Efficient Integration of a realistic Two-dimensional Cardiac Tissue Model by Domain Decomposition. IEEE Trans. Biomed. Eng. 45, 372-384 (1998)

11. Panfilov, A.V.: Three-dimensional organization of electrical turbulence in the heart. Phys. Rev. E 59, R6251-R6254 (1999)

12. Szilágyi, S.M., Szilágyi, L., Benyó, Z.: Spatial Heart Simulation and Analysis Using Unified Neural Network. Ser. Adv. Soft Comput. 41, 346-354 (2007)

13. ten Tusscher, K.H.W.J., Bernus, O., Hren, R., Panfilov, A.V.: Comparison of electrophysiological models for human ventricular cells and tissues. Prog. Biophys. Mol. Biol. 90, 326-345 (2006)

14. ten Tusscher, K.H.W.J., Noble, D., Noble, P.J., Panfilov, A.V.: A model for human ventricular tissue. Amer. J. Physiol. Heart. Circ. Physiol. 286, H1573-H1589 (2004)

15. Winfree, A.T.: Electrical turbulence in three-dimensional heart muscle. Science 266, 1003-1006 (1994)

16. Winslow, R.L., Hinch, R., Greenstein, J.L.: ICCS 2000. Lect. Notes Math, vol. 1867, pp. $97-131$ (2005)

17. Winslow, R.L., Scollan, D.F., Holmes, A., Yung, C.K., Zhang, J., Jafri, M.S.: Electrophysiological Modeling of Cardiac Ventricular Function: From Cell to Organ. Ann. Rev. Biomed Eng. 2, 119-155 (2000) 